\title{
PÉRDIDAS AUDITIVAS RELACIONADAS CON LA EXPOSICIÓN A RUIDO EN TRABAJADORES DE LA CONSTRUCCIÓN
}

\author{
HEARING LOSS RELATED WITH NOISE EXPOSURE IN CONSTRUCTION WORKERS
}

\author{
PILAR GÓMEZ MUR(1), BRÍGIDO PÉREZ BERMÚDEZ(1), ALFONSO MENESES MONROY(1) \\ (1) Área de Salud Laboral de MEDYCSA, Servicio de Prevención Ajeno. Madrid. España
}

\section{RESUMEN}

INTRODUCCIÓN: A pesar del ruido existente en las obras de la construcción no está extendido el uso de protectores auditivos en este sector.

OBJETIVO: Estudiar las pérdidas auditivas inducidas por ruido (PAIR) en las audiometrías de estos trabajadores.

MATERIAL Y MÉTODOS: Se realiza un estudio descriptivo de las audiometrías de tonos puros realizadas a 223 trabajadores del sector y se comparan con las de 262 administrativos. Para clasificar las PAIR se ha utilizado el método desarrollado por Klockhoff $(\mathrm{K})$ y modificado por la clínica del Lavoro de Milan. Se analiza también la presencia de escotomas en las frecuencias 3000, 4000 ó 6000 $\mathrm{Hz}$.

RESULTADOS: La prevalencia de PAIR y escotomas bilaterales ha sido significativamente superior en el grupo de la construcción (K: $\mathrm{OR}=3,1$. IC95\%:1,801-5,435. Escotoma audiométrico bilateral: $\mathrm{OR}=3,8$. IC95\%:2.2446.606). Aunque el escotoma en $6000 \mathrm{~Hz}$ ha sido el más frecuente, la diferencia entre los dos grupos únicamente ha sido significativa en el de $4000 \mathrm{~Hz}$.

DISCUSIÓN: Entre un 20,7 (K) y un 24,3\% (escotomas) de los trabajadores de la construcción presentan PAIR bilaterales ( 8,4 y $8,8 \%$ en administrativos). Es necesario incidir en las medidas preventivas, fundamentalmente en el uso de protectores auditivos adecuados. Se recomienda profundizar en la etiología de los diferentes escotomas en futuros estudios.

Palabras clave: Exposición a ruido, trabajadores de la construcción, pérdida auditiva inducida por ruido, escotoma audiométrico, medidas preventivas.

(Med Segur Trab 2008; 54 (213):33-40)

\section{ABSTRACT}

INTRODUCTION: Despite the noise produced in constructions works, the utilization of hearing protection devices is not common in this sector.

AIM: To study the noise induced hearing loss (NIHL) present on the audiometries of the construction workers.

METHODOLOGY: A descriptive study is made on the pure tone audiometries done to 223 construction workers, comparing them with those coming from 262 administrative ones. For audiometries classification purposes, the method developed by Klockhoff $(\mathrm{K})$ and modified by Clinica del Lavoro in Milan, is used. Presence of a notch in 3.000, 4.000 or $6.000 \mathrm{~Hz}$ frequencies is also analyzed.

RESULTS: A significantly higher NIHL bilateral prevalence has been found in the constructions group (K: $\mathrm{OR}=3,1$. IC95\%:1,801-5,435. Bilateral notch: $\mathrm{OR}=3,8$. IC95\%:2.244-6.606).) Although the $6.000 \mathrm{~Hz}$ notch is the most frequent, the difference between both groups is only significant for the $4.000 \mathrm{~Hz}$ one .

DISCUSION: Between $20.7 \%$ and $24.3 \%$ of construction workers present bilateral NIHL $(8.4 \%$ and $8.8 \%$ in administrative ones). It is necessary to reinforce the utilization of preventive measures, specially hearing protection devices. It is recommended to go more deeply into different notches etiology in future studies.

Key words: Noise exposure, construction works, noise induced hearing loss, audiometric notch, preventive measures.

(Med Segur Trab 2008; 54 (213):33-40)

\section{Correspondencia:}

Dra. Pilar Gómez Mur

Área de Salud Laboral de Medycsa

Valentín Beato, 7. Edificio Medycsa

28027 Madrid (España)

Teléfono: 913751811

pgomez@medycsa.com 


\section{INTRODUCCIÓN}

Aunque existen referencias suficientes para afirmar que los trabajadores de la construcción están expuestos a niveles nocivos de ruido $(1,2,3)$, en nuestro país no está extendida la idea de que deba ser obligatorio el uso de protectores auditivos en las obras. En las evaluaciones de riesgos consultadas de este sector, realizadas por diferentes Servicios de Prevención, es infrecuente encontrar evaluaciones específicas de ruido con mediciones del nivel diario equivalente al que están expuestos los trabajadores. La rotación de puestos de trabajo, la temporalidad en el sector, la variabilidad de tareas, etc., son factores que dificultan estas mediciones.

Según recoge la VI Encuesta Nacional de Condiciones de Trabajo (4) el 21,9\% de los trabajadores de la construcción manifestaron estar expuestos a niveles de ruido elevados o muy elevados. No hemos encontrado estudios publicados en España referentes a las pérdidas auditivas inducidas por ruido (PAIR) en la construcción.

En el cuadro de enfermedades profesionales listado en el Real Decreto 1299/2006, de 10 de diciembre (5), la hipoacusia producida por el ruido laboral se encuadra entre las causadas por agentes físicos y se define como "sordera de tipo neurosensorial, frecuencias de 3 a $6 \mathrm{KHz}$, bilateral, simétrica e irreversible", producida en "trabajos que exponen a ruidos continuos cuyo nivel sonoro diario equivalente (según legislación vigente) sea igual o superior a 80 decibelios A". Entre los trabajos que menciona que exponen a los trabajadores a ruidos continuos cuyo nivel sonoro diario equivalente sea igual o superior a $80 \mathrm{~dB} \mathrm{~A}$, encontramos los siguientes pertenecientes a la construcción:

- Trabajos con martillos y perforadores neumáticos en minas, túneles y galerías subterráneas.

- Trabajos de obras públicas (rutas, construcciones, etc.) efectuados con máquinas ruidosas, como las bulldozers, excavadoras, palas mecánicas, etc.

No se pone en duda que en los trabajos citados pueda generarse mucho ruido, sin embargo, incluso en profesiones en las que aparentemente los niveles de ruido no parecen ser muy elevados, como el caso de los electricistas, trabajos como el de Seixas y colaboradores (3) han demostrado la existencia de niveles potencialmente dañinos.

Varios estudios consultados $(1,2,6)$ y la obser- vación de nuestros técnicos de Prevención en su práctica diaria, muestran el escaso uso que estos trabajadores hacen de los protectores auditivos. Entre las posibles causas podemos citar: la necesidad de escuchar en las obras señales acústicas de aviso o alarma, el no disponer de los protectores, que les incomode su uso, que sus responsables no incidan en esta medida preventiva, etc.

Consideramos de interés estudiar y poner de manifiesto las PAIR existentes en los trabajadores de la construcción para fomentar la adopción de medidas preventivas eficaces, especialmente entre los empresarios y trabajadores del sector.

\section{METODOLOGÍA}

Se ha realizado un estudio transversal en el que se describen los resultados de las audiometrías de tonos puros realizadas a un grupo de 223 trabajadores de la construcción, y se comparan con las realizadas a un grupo 262 trabajadores no expuestos a ruido (administrativos).

En los dos grupos se excluyó inicialmente del estudio a los que presentaban patología aguda de oído, tapones de cerumen o hipoacusia de causa conocida diferente a la laboral, también a los que habían estado sometidos a ruido en oficios anteriores no pertenecientes al sector objeto del estudio. Todos los trabajadores incluidos en el estudio fueron varones. En el grupo de trabajadores de la construcción se incluyeron prácticamente todos los oficios del sector, salvo los mencionados en el RD 1299/2006 (5): conductores de maquinaria ruidosa y los trabajadores que manejan martillos neumáticos.

A los trabajadores de los dos grupos que acudieron a la Clínica Príncipe de Vergara de Medycsa, Servicio de Prevención Ajeno, para hacerse un examen periódico de Vigilancia de la Salud, se les realizó una audiometría tonal por vía aérea. El marco temporal del estudio fueron los cuatro primeros meses del año 2007. El muestreo fue no probabilístico consecutivo.

Las audiometrías fueron realizadas en cabina, por una enfermera de empresa con varios años de experiencia en la realización de esta prueba. Se verificó la calibración periódica del audiómetro utilizado, según los criterios de la norma UNE-EN ISO 389-1:2001.

En el grupo de la construcción se recogieron las siguientes variables: edad, exposición a ruido extralaboral, antecedentes familiares de sordera, antece- 
dentes patológicos (diabetes e hipertensión), tiempo en el sector, oficio, uso de disolventes en el trabajo y utilización de protectores auditivos. En 105 de los 262 trabajadores administrativos se recogió: edad, exposición a ruido extralaboral, antecedentes familiares de sordera y antecedentes patológicos (diabetes e hipertensión). En los 157 administrativos restantes únicamente la edad y los resultados de la audiometría.

Se han presentado los resultados de las audiometrías de la siguiente manera:

- Clasificados por el método Klockhoff.

- Según la existencia o no un escotoma en las frecuencias 3000 ó 4000 ó $6000 \mathrm{~Hz}$.
Para clasificar las pérdidas auditivas inducidas por ruido (PAIR) se ha utilizado el método desarrollado por Klockhoff y modificado por la clínica del Lavoro de Milan (7). Este método, ampliamente utilizado, es uno de los citados en el Protocolo de ruido del Ministerio de Sanidad y Consumo (8) para clasificar las audiometrías de los trabajadores expuestos a ruido y ha sido recomendado, entre otros, por Sánchez Galán y Rodríguez Ortiz de Salazar (9) para evaluar la hipoacusia profesional. Resulta en la práctica un método simple y práctico para la diferenciación entre la normalidad y los hallazgos patológicos compatibles o no con la exposición a ruido. Se tienen en cuenta las pérdidas en frecuencias conversacionales $(500-3000 \mathrm{~Hz})$ y la presencia o no de trauma acústico en altas frecuencias (4000-8000). Se ha considerado trauma acústico la presencia de un

Tabla I. PAIR (método Klockhoff) y escotomas bilaterales en altas frecuencias en trabajadores de la construcción, relacionados con las diferentes variables recogidas en el estudio.

\begin{tabular}{|c|c|c|c|}
\hline VARIABLES & PAIR (método Klockhoff) & No PAIR (método Klockhoff) & $\mathbf{p}$ \\
\hline Edad (años) & $41.7(9.7)^{* *}$ & $37.7(11.8)^{* *}$ & 0.019 \\
\hline $\begin{array}{l}\text { Tiempo en el } \\
\text { sector (años) }\end{array}$ & $16.6(12.3)^{* *}$ & $13.2(11.7)^{* *}$ & 0.098 \\
\hline Hipertensión & $2(4.5 \%)$ & $11(6.5 \%)$ & 0.622 \\
\hline Diabetes & $0(0.0 \%)$ & $4(2.4 \%)$ & $0.582 *$ \\
\hline $\begin{array}{l}\text { Exposición a ruido } \\
\text { extralaboral }\end{array}$ & $8(20.5 \%)$ & $24(15.1 \%)$ & 0.410 \\
\hline Disolventes & $15(34.9 \%)$ & $45(27.3 \%)$ & 0.327 \\
\hline $\begin{array}{l}\text { Antecedente } \\
\text { familiar de } \\
\text { hipoacusia }\end{array}$ & $7(18.4 \%)$ & $19(12.6 \%)$ & 0.428 \\
\hline \multirow{2}{*}{$\begin{array}{l}\text { Uso habitual de } \\
\text { protectores } \\
\text { auditivos }\end{array}$} & $3(6,7 \%)$ & $10(5,8 \%)$ & 0.391 \\
\hline & $\begin{array}{l}\text { Escotoma bilateral en altas } \\
\text { frecuencias }\end{array}$ & $\begin{array}{l}\text { Sin escotoma bilateral en altas } \\
\text { frecuencias }\end{array}$ & \\
\hline Edad (años) & $43.4(10.2)^{* *}$ & $37.0(11.5) * *$ & 0.000 \\
\hline $\begin{array}{l}\text { Tiempo en el } \\
\text { sector (años) }\end{array}$ & $18.0(12.6) * *$ & $12.6(11.4)^{* *}$ & 0.005 \\
\hline Hipertensión & $4(7.8 \%)$ & $9(5.6 \%)$ & 0.559 \\
\hline Diabetes & $2(3.9 \%)$ & $2(1.2 \%)$ & $0.245 *$ \\
\hline $\begin{array}{l}\text { Exposición a ruido } \\
\text { extralaboral }\end{array}$ & $9(19.6 \%)$ & $23(15.1 \%)$ & 0.474 \\
\hline $\begin{array}{l}\text { Disolventes } \\
\text { (laboral) }\end{array}$ & $17(33.3 \%)$ & $43(27.4 \%)$ & 0.416 \\
\hline $\begin{array}{l}\text { Antecedente } \\
\text { familiar de } \\
\text { hipoacusia }\end{array}$ & $8(17.8 \%)$ & $18(12.5 \%)$ & 0.370 \\
\hline $\begin{array}{l}\text { Uso habitual de } \\
\text { protectores } \\
\text { auditivos }\end{array}$ & $4(7,5 \%)$ & $9(5,5 \%)$ & 0.797 \\
\hline
\end{tabular}


escotoma o muesca (notch en inglés) en las frecuencias 4000-6000 Hz, con recuperación en $8000 \mathrm{~Hz}$. No se lleva a cabo ninguna corrección por la edad.

Se han mostrado también los resultados de las audiometrías según exista o no un escotoma en altas frecuencias (3000 ó 4000 ó $6000 \mathrm{~Hz}$ ). En varios trabajos sobre pérdidas auditivas y exposición a ruido $(10,11,12)$ se han encontrado escotomas en estas tres frecuencias, aunque sea la de $4000 \mathrm{~Hz}$ la principalmente afectada (8). El Real Decreto 1299/2006 (5) menciona expresamente, en la definición de hipoacusia por ruido, las frecuencias $3000,4000 \mathrm{y}$ $6000 \mathrm{~Hz}$.

En este trabajo se ha considerado escotoma en altas frecuencias la existencia en la audiometría de un valor a partir de $30 \mathrm{~dB}$ (inclusive) en las frecuencias 3000 ó 4000 ó $6000 \mathrm{~Hz}$, teniendo un valor menor, en al menos $5 \mathrm{~dB}$, las dos frecuencias adyacentes. Se ha considerado también que existe un escotoma en los casos en los que han sido dos las frecuencias afectadas $(3000-4000 \mathrm{~Hz}$ ó 4000-6000 $\mathrm{Hz}$ ), existiendo recuperación en las adyacentes.

De acuerdo con la definición de la hipoacusia por ruido en el RD 1299/2006 como bilateral y simétrica (5), en este estudio se ha incidido en los hallazgos bilaterales, aunque se han reflejado también los unilaterales. No se ha tenido en cuenta específicamente la simetría de las gráficas en ambos oídos.

Para el análisis de los datos se ha utilizado el programa estadístico SPSS, versión 16.0. Para las proporciones se ha utilizado el ji cuadrado y para las variables cuantitativas la T-Student. Significación $\mathrm{p}<0.05$. Para ajustar por la edad se construye un modelo de Regresión Logística no condicional, cuya medida de asociación es la Odds Ratio (O.R) con sus intervalos de confianza al $95 \%$.

\section{RESULTADOS}

Se muestran a continuación los resultados de determinadas variables recogidas en los trabajadores de la construcción en relación con la existencia o no de hallazgos compatibles con la alteración al ruido: PAIR bilateral (método Klockhoff) y presencia de escotomas bilaterales en altas frecuencias.

Al comparar los hallazgos encontrados en los 223 trabajadores de la construcción con los 262 administrativos se observan los siguientes resultados (Tabla II)
Al comparar los dos grupos de trabajadores se observa que existen diferencias significativas en la edad, siendo el grupo de la construcción más joven, por lo que se construyeron dos modelos de Regresión Logística para ajustarla por dicha variable.

Modelo 1: la variable dependiente es PAIR bilateral clasificada por el método Klockhoff, siendo las variables independientes el sector (trabajadores de la construcción /administrativos) y la edad. En el Modelo 2 la variable dependiente es escotoma bilateral en altas frecuencias y las variables independientes las mismas, sector y edad.

Como puede verse en la tabla III existe una OR 3.1 veces más de PAIR bilateral diagnosticada por el método Klockhoff en la construcción que en trabajadores administrativos. Dicha asociación está controlada (ajustada) por la edad.

La curva ROC de los valores predichos es de $69.3(61.9-74,6)$.

Como puede verse en la tabla IV existe una OR 3.8 veces más de escotomas bilaterales en altas frecuencias en la construcción que en trabajadores administrativos e igualmente dicha asociación está controlada por la edad.

La curva ROC de los valores predichos es de $71.2(64.5-77.3)$.

Tanto en la clasificación por el método Klockhoff como por la presencia de escotomas en altas frecuencias, independientemente de la edad, se observan más de 3 veces más hallazgos compatibles con la exposición a ruido en la construcción que en los trabajadores administrativos.

Se desglosan en la tabla $\mathrm{V}$ los escotomas según la frecuencia (en $\mathrm{Hz}$ ) en la que se presentan, comparando los hallazgos de la construcción con los de los administrativos.

Atendiendo concretamente al oído que se afecta con más frecuencia entre los trabajadores de la construcción observamos en la siguiente tabla que el oído izquierdo es el más afectado, no siendo la diferencia estadísticamente significativa.

\section{DISCUSIÓN}

Los hallazgos patológicos compatibles con la exposición a ruido han sido más frecuentes en el sector de la construcción (ver Tabla II), siendo la dife- 
Tabla II. Comparación de los resultados de las variables recogidas y de los hallazgos de las audiometrías entre los dos grupos.

\begin{tabular}{|l|l|l|l|}
\hline VARIABLES & CONSTRUCCION & ADMINISTRATIVO & P \\
\hline Edad (años) & $38.6(11.5)$ & $41.6(10.1)$ & $\mathbf{0 . 0 0 3}$ \\
\hline Hipertensión & $13(6.1 \%)$ & $8(7.6 \%)$ & 0.635 \\
\hline Diabetes & $4(1.9 \%)$ & $3(2.9 \%)$ & 0.688 \\
\hline Exposición a ruido extralaboral & $32(16.1 \%)$ & $12(11.4 \%)$ & 0.307 \\
\hline $\begin{array}{l}\text { Antecedente familiar de } \\
\text { hipoacusia }\end{array}$ & $26(13.7 \%)$ & $21(20 \%)$ & 0.184 \\
\hline $\begin{array}{l}\text { Escotoma derecho en altas } \\
\text { frecuencias }\end{array}$ & $83(36,9 \%)$ & $60(22,9 \%)$ & $\mathbf{0 . 0 0 4}$ \\
\hline $\begin{array}{l}\text { Escotoma izquierdo en altas } \\
\text { frecuencias }\end{array}$ & $97(43,5 \%)$ & $58(22,1 \%)$ & $\mathbf{0 . 0 0 0}$ \\
\hline $\begin{array}{l}\text { Escotoma bilateral en altas } \\
\text { frecuencias }\end{array}$ & $54(24.3 \%)$ & $23(8.8 \%)$ & $\mathbf{0 . 0 0 0}$ \\
\hline Klockhof derecho & & & $\mathbf{0 . 0 0 7}$ \\
\hline Normal & $90(40.4 \%)$ & $136(51.9 \%)$ & \\
\hline Trauma leve & $44(19.7 \%)$ & $40(15.3 \%)$ & \\
\hline Trauma avanzado & $4(1.8 \%)$ & $0(0 \%)$ & \\
\hline Hipoacusia leve por ruido & $21(9.4 \%)$ & $9(3.4 \%)$ & \\
\hline Hipoacusia Moderada por ruido & $4(1.8 \%)$ & $3(1.1 \%)$ & \\
\hline Hipoacusia avanzada por ruido & $0(0 \%)$ & $1(0.4 \%)$ & \\
\hline Alteración no debida a ruido & $60(26.9 \%)$ & $73(27.9 \%)$ & \\
\hline Klockhoff izquierdo & & & $\mathbf{0 . 0 0 0}$ \\
\hline Normal & $95(42.8 \%)$ & $131(50 \%)$ & \\
\hline Trauma leve & $50(22.5 \%)$ & $39(14.9 \%)$ & \\
\hline Trauma avanzado & $5(2.3 \%)$ & $4(1.5 \%)$ & \\
\hline Hipoacusia leve por ruido & $29(13.1 \%)$ & $11(4.2 \%)$ & \\
\hline Hipoacusia moderada por ruido & $2(0.9 \%)$ & $1(0.4 \%)$ & \\
\hline Hipoacusia avanzada por ruido & $3(1.4 \%)$ & $0(0 \%)$ & \\
\hline Alteración no debida a ruido & $38(17.1 \%)$ & $76(29 \%)$ & \\
\hline Klockhoff: PAIR bilateral & $46(20.7 \%)$ & $22(8.4 \%)$ & \\
\hline & & & \\
\hline
\end{tabular}

(*) = Media (Desviación Típica)

rencia entre los dos grupos significativa. Los resultados de este estudio muestran que los trabajadores del grupo de la construcción presentan 3,1 veces más pérdidas auditivas inducidas por ruido bilaterales clasificadas por el método Klockhoff, que los administrativos, y 3,8 veces más escotomas bilaterales en 3000 ó 4000 ó 6000 Hz (ver Tablas III y IV). También se han encontrado diferencias estadísticamente significativas considerando cada oído por separado, siendo siempre más frecuentes los hallazgos patológicos en el sector de la construcción (ver Tabla II).

Como puede verse en la tabla I, en los trabajadores de la construcción existe una asociación entre la edad de los trabajadores y el tiempo que llevan trabajando en el sector, y la presencia de hallazgos bilaterales compatibles con la exposición a ruido. A mayor edad y más años en el sector mayor presencia de PAIR y escotomas bilaterales en altas frecuencias.
El hecho de que en más de la mitad de los trabajadores administrativos no se disponga de información sobre factores como exposición extralaboral a ruido y antecedentes familiares de sordera constituye una limitación y, en principio, puede sesgar este estudio. Esta circunstancia parece poco relevante ya que, considerando los 105 administrativos en los que sí se dispone de esta información, no existen diferencias significativas, respecto a estas variables, con el grupo de los trabajadores de la construcción. Por otro lado entre los trabajadores de la construcción no se han encontrado, en cuanto a las PAIR (método K) y escotomas bilaterales, diferencias significativas entre los que referían exposición extralaboral y los que no. Hay que tener en cuenta que en este trabajo los datos sobre exposición extralaboral a ruido son subjetivos y podría existir un sesgo en la información por parte de los trabajadores. Aunque la exposición extralaboral a ruido es muy difícil de 
Tabla III. Modelo 1 de Regresión Logística cuya variable dependiente es la PAIR bilateral (método Klockhoff) y las variables independientes son el sector y la edad.

\begin{tabular}{|l|l|l|}
\hline Variables en ecuación & Odds Ratio & Intervalo confianza 95 \% \\
\hline Construcción & 3.129 & $(1.801-5.435)$ \\
\hline Edad & 1.028 & $(1.004-1.053)$ \\
\hline
\end{tabular}

Tabla IV. Modelo 2 de Regresión Logística cuya variable dependiente es el escotoma bilateral en altas frecuencias y las independientes el sector y la edad.

\begin{tabular}{|l|l|l|}
\hline Variables en ecuación & Odds Ratio & Intervalo confianza 95\% \\
\hline Construcción & 3.851 & $(2.244-6.606)$ \\
\hline Edad & 1.041 & $(1.018-1.065)$ \\
\hline
\end{tabular}

cuantificar, Neitzel y colaboradores (13) estimaron de una manera objetiva la exposición fuera del trabajo en trabajadores de la construcción. Obtuvieron datos curiosos como los que hacían uso de las armas de fuego con fines recreativos, incluso sin considerar el uso de las mismas, estaban más expuestos a ruido extralaboral que los que no las usaban, explicando esta circunstancia el hecho de que participaban más en otras actividades recreativas con exposición a ruido. Encontraron que un $20 \%$ de los trabajadores estudiados tenía una exposición extralaboral que podía suponer un riesgo de pérdida auditiva.

Constituye otra limitación para este estudio que las audiometrías se hayan realizado exclusivamente por vía aérea, no obstante, dado el tamaño de la muestra, se asume que alguna de las hipoacusias consideradas como compatibles con la exposición a ruido pueda ser de transmisión.
Se han encontrado en este estudio escotomas bilaterales en altas frecuencias en un $24,3 \%$ de los trabajadores de la construcción y en un $8,8 \%$ de los administrativos. Muchos más si tenemos en cuenta cada oído por separado (ver tabla II). Aunque, como puede verse en la Tabla V, el escotoma más prevalente en ambos grupos ha sido el de la frecuencia $6000 \mathrm{~Hz}$, es en el de la frecuencia $4000 \mathrm{~Hz}$, en el que la diferencia ha resultado estadísticamente significativa. No encontramos una explicación satisfactoria para la elevada prevalencia de escotomas encontrados en la frecuencia $6000 \mathrm{~Hz}$, en trabajadores de los dos grupos.

Hay que tener en cuenta que no existe una definición estandarizada del término escotoma por lo que cada profesional tiende a desarrollar sus propios criterios. El trabajo de Rabinowitz y colaboradores (10), grupo perteneciente al Programa de Medicina Laboral y Medioambiental de la Universidad de

Tabla V. Comparación entre la frecuencia de presentación de los diferentes escotomas en altas frecuencias en los dos grupos.

\begin{tabular}{|l|l|l|c|}
\hline VARIABLES & CONSTRUCCION & ADMINISTRATIVO & P \\
\hline Escotoma derecho & & & \\
\hline Sin Escotoma & $140(63.1 \%)$ & $202(77.1 \%)$ & 0,001 \\
\hline Escotoma en 3000 & $10(4.5 \%)$ & $6(2.3 \%)$ & 0,175 \\
\hline Escotoma en 4000 & $29(13.1 \%)$ & $13(5 \%)$ & $\mathbf{0 . 0 0 0}$ \\
\hline Escotoma en 6000 & $32(14.4 \%)$ & $32(12.2 \%)$ & 0,476 \\
\hline Escotoma en 3-4000 o 4-6000 & $11(5.0 \%)$ & $9(3.4 \%)$ & 0,403 \\
\hline Escotoma izquierdo & & & \\
\hline Sin Escotoma & $126(56.5 \%)$ & $204(77.9 \%)$ & 0,000 \\
\hline Escotoma en 3000 & $7(3.1 \%)$ & $3(1.1 \%)$ & 0,198 \\
\hline Escotoma en 4000 & $34(15.2 \%)$ & $14(5.3 \%)$ & $\mathbf{0 . 0 0 0}$ \\
\hline Escotoma en 6000 & $43(19.3 \%)$ & $35(13.4 \%)$ & 0,077 \\
\hline Escotoma en 3-4000 o 4-6000 & $13(5.8 \%)$ & $6(2.3 \%)$ & $\mathbf{0 , 0 4 5}$ \\
\hline Escotoma bilateral & $54(24.3 \%)$ & $23(8.8 \%)$ & $\mathbf{0 . 0 0 0}$ \\
\hline
\end{tabular}


Yale, mostró consenso en un grupo de expertos a la hora de diagnosticarlo. Por el contrario Mc Bride y Williams (11) observaron, en un trabajo sobre los escotomas audiométricos como signo de la pérdida auditiva inducida por ruido, que existía una gran variabilidad en la detección de los mismos en un grupo de tres expertos

Como se refleja en libros de texto de hace más de 30 años (14) y en múltiples estudios, la frecuencia de $4000 \mathrm{~Hz}$ es la más precozmente afectada por la exposición a ruido. McBride y Williams (11) estudiaron los escotomas en trabajadores expuestos a diferentes tipos de ruido y encontraron también una mayor prevalencia de escotomas en la frecuencia $6000 \mathrm{~Hz}$, que no mostraban asociación con la exposición, sí encontraron esta asociación en el escotoma de la frecuencia $4000 \mathrm{~Hz}$. Celik y colaboradores (15) utilizando emisiones otoacústicas compararon un grupo de expuestos a ruido con un grupo control, encontrando diferencias estadísticamente significativas en $4000 \mathrm{~Hz}$ y no en $6000 \mathrm{~Hz}$. Rainal y colaboradores (16) estudiando a un grupo de pilotos militares, encontraron los escotomas en 3000 y 6000 $\mathrm{KHz}$, según se tratara de pilotos de helicóptero o de transporte, respectivamente.
Se ha encontrado en este estudio, en los trabajadores de la construcción, una mayor prevalencia de hallazgos compatibles con la exposición a ruido en el oído izquierdo que en el derecho, aunque la diferencia no ha sido estadísticamente significativa (ver Tabla VI). Dado que la exposición a ruido en las obras no tiene porqué ser unilateral, no encontramos una explicación plausible para esta lateralización que también reflejan otros estudios $(16,17)$ el de Raynal y colaboradores en un grupo muy diferente, pilotos militares. En algunos de casos quizás podría estar relacionada con la conducción de vehículos ya que la pérdida predominante en oído izquierdo es característica de la hipoacusia del conductor.

Independientemente de lo anteriormente citado sobre las características de la PAIR, y, concretamente de los escotomas, aspecto que requiere un análisis más profundo y abre expectativas para nuevos estudios en este campo, más de un $20 \%$ de los trabajadores de la construcción presentan hallazgos bilaterales compatibles con la exposición a ruido. $\mathrm{La}$ mayor parte de estos hallazgos han sido traumas leves e hipoacusias leves (ver Tabla II).

La adopción de medidas preventivas eficaces en el lugar de trabajo puede evitar la progresión de estas

Tabla VI. Comparación de la frecuencia de presentación de hallazgos compatibles con la exposición a ruido en cada uno de los oídos, en el grupo de la construcción.

\begin{tabular}{|l|l|l|l|}
\hline & Oído Dcho. & Oído Izq. & p \\
\hline Método KLOCKHOFF & & & 0,107 \\
\hline Normal o hipoacusia no debida a ruido & $150(67.3 \%)$ & $133(59.9 \%)$ & \\
\hline Hipoacusia por ruido & $73(32.7 \%)$ & $89(40.1 \%)$ & \\
\hline ESCOTOMA en altas frecuencias & & & 0.158 \\
\hline Ausencia de escotoma & $140(63.1 \%)$ & $126(56.5 \%)$ & \\
\hline Presencia de escotoma & $82(36.9 \%)$ & $97(43.5 \%)$ & \\
\hline
\end{tabular}

pérdidas y que la vida social del trabajador se vea afectada. Teniendo en cuenta siempre los criterios del Real Decreto 286/2006 (18), podría plantearse la adquisición de máquinas menos ruidosas o medidas administrativas que redujeran el tiempo de exposición, aunque siendo realistas, por las características del trabajo en este sector, el uso de protectores auditivos adecuados (solo un 5,9\% de la muestra estudiada los usan habitualmente) es la medida fundamental y debería promoverse, e informar y formar adecuadamente a los trabajadores para fomentar su uso adecuado. Estos protectores deben permitir escuchar las señales de aviso y alarma. Desde el punto de vista de la Medicina del Trabajo deben llevarse a cabo controles periódicos de la audición, preferentemente anuales, incidiendo en la detección precoz de las PAIR y en la comunicación de sospecha de enfermedad profesional, si procede. Recomendar al trabajador que evite la exposición a ruido extralaboral y la toma de medicamentos ototóxicos, y valorar una exposición concomitante a agentes químicos tóxicos para el oído pueden ser también medidas preventivas eficaces. 


\section{AGRADECIMIENTOS}

Agradecemos a Montserrat Noriega de Francisco, Directora de Salud Laboral de MEDYCSA y a Esther Díaz Gigorro, Directora de la clínica Príncipe de Vergara de la misma entidad, el habernos facilitado la obtención de los datos para la realización del estudio.

\section{$\overline{\text { BIBLIOGRAFÍA }}$}

1. Suter AH. Construction Noise: Exposure, Effects and the potencial for Remediation; A review and analysis. Am Ind Hyg Assoc J. 2002 NovDec;63(6):768-89.

2. Neitzel R, Seixas N. The efectiveness of Hearing Protection Among Construction Workers. Occup Environ Hyg. 2005 Apr;2(4):227-38

3. Seixas NS, Ren K, Neitzel R, Camp J, Yost M. Noise exposure among construction electricians. Am Ind Hyg Assoc J. 2001 Sep-Oct;62(5):615-21

4. Instituto Nacional de Seguridad e Higiene en el Trabajo. VI Encuesta Nacional de Condiciones de Trabajo (online). 2007 (citado 22 septiembre 2008), p 63-4 . Disponible en: http://www.oect.es/ Observatorio/Contenidos/InformesPropios/Desarrollados/Fi cheros/Informe_VI_ENCT.pdf

5. Real Decreto 1299/2006, de 10 de noviembre, por el que se aprueba el cuadro de enfermedades profesionales en el sistema de la Seguridad Social y se establecen criterios para su notificación y registro. Ministerio de Trabajo e Inmigración. BOE de 19/12/2006, núm 302. 44487-44546.

6. Lusk SL, Kerr MJ, Kauffman SA. Use of hearing protection and perceptions of noise exposure and hearing loss among construction workers. Am Ind Hyg Assoc J. 1998 Jul;59(7):466-70

7. Merluzzi F, Cornacchia L, Parigi G, Terrana T. Metodología di esecuzione del controllo dell'udito dei lavoratori esposti al rumore, Arch. Otol., 1979; 4: 695-714.

8. Comisión de Salud Pública. Consejo Interterritorial del Sistema Nacional de Salud. Ruido. Protocolos de Vigilancia Sanitaria Específica. Ministerio de Sanidad y Consumo; 2000.

9. Sánchez Galán L, Rodríguez Ortiz de Salazar B. Revisión médico-legal y estado actual de la eva-
Así mismo agradecemos a Fuencisla Sanz Luengo, Directora de la Biblioteca de la Escuela Universitaria de Enfermería, Fisioterapia y Podología de la Universidad Complutense de Madrid, la aportación de los artículos seleccionados para el estudio.

luación médica de la hipoacusia profesional en el sistema español de la Seguridad Social. Med Segur Trab 2005; Vol LI n ${ }^{\circ}$ 198: 7-20.

10. Rabinowitz PM, Galusha D., Slade MD, Dixon-Ernst C, Sircar KD, Dobie RA. Audiogram notches in noise-exposed workers. Ear Hear. 2006 Dec;27(6):742- 50.

11. Mc Bride D I, Williams S. Audiometric notch as a sign of noise induced hearing loss. Occup. Environ. Med. 2001;58:46-51

12. Seixas NS, Goldman B, Sheppard L, Neitzel R, Norton S, Kujawa SG. Prospective noise induced changes to hearing among construction industry apprentices. Occup Environ Med. 2005 May;62(5):309-17.

13. Neitzel R, Seixas N, Goldman B, Daniell W. Contributions of Non-occupational activities to total noise exposure of construction workers. Ann occup Hyg. 2004;48(5):463 - 73.

14. Fairen M. Oido. Temas de O.R.L. Zaragoza: Editorial Pórtico; 1977. p 423-60.

15. Celik O, Yalcin S, Ortürk A. Hearing parameters in noise exposed industrial workers. Auris Nasus Larynx. 1998 Dec; 25(4): 369-75

16. Raynal M, Kossowski M, Job A. Hearing in military pilots: one-time audiometry in pilots of fighters, transports, and helicopters. Aviat Space Environ Med. 2006 Jan;77(1):57-61

17. Hong O. Hearing loss among operating engineers in American construction industry. Int Arch Occup Environ Health. 2005 Aug;78(7):565-74

18. Real Decreto 286/2006, de 10 de marzo, sobre la protección de la salud y la seguridad de los trabajadores contra los riesgos relacionados con la exposición al ruido. BOE de 11/03/2006, núm. 60 . 9842-9848. 